\title{
HispanismeS
}

Revue de la Société des Hispanistes Français

$17 \mid 2021$

Murs, barrières, obstacles dans les mondes

hispaniques I

\section{Les frontières de Willy ou l'itinéraire heurté de Casper le marero dans le film Sin Nombre de Cary Joji Fukunaga (2009)}

Las fronteras de Willy o el accidentado itinerario del pandillero Casper en la película Sin Nombre (2009) de Cary Joji Fukunaga

The boundaries of Willy or the colliding itinerary of Casper the marero in Cary Joji Funkunaga's Sin Nombre (2009)

\section{Safia Boufalaas}

\section{OpenEdition}

\section{Journals}

Édition électronique

URL : https://journals.openedition.org/hispanismes/13413

DOI : 10.4000/hispanismes.13413

ISSN : 2270-0765

\section{Éditeur}

Société des Hispanistes Français

\section{Référence électronique}

Safia Boufalaas, «Les frontières de Willy ou l'itinéraire heurté de Casper le marero dans le film Sin Nombre de Cary Joji Fukunaga (2009) », HispanismeS [En ligne], 17 | 2021, mis en ligne le 01 juin 2021, consulté le 27 janvier 2022. URL : http://journals.openedition.org/hispanismes/13413; DOI : https:// doi.org/10.4000/hispanismes. 13413

Ce document a été généré automatiquement le 27 janvier 2022.

\section{cc) $(\mathrm{S})$}

Les contenus de cette revue sont mis à disposition selon les termes de la Licence Creative Commons Attribution - Pas d'Utilisation Commerciale - Pas de Modification 4.0 International. 


\title{
Les frontières de Willy ou l'itinéraire heurté de Casper le marero dans le film Sin Nombre de Cary Joji Fukunaga (2009)
}

\author{
Las fronteras de Willy o el accidentado itinerario del pandillero Casper en la \\ película Sin Nombre (2009) de Cary Joji Fukunaga \\ The boundaries of Willy or the colliding itinerary of Casper the marero in Cary \\ Joji Funkunaga's Sin Nombre (2009)
}

Safia Boufalaas

\section{Introduction}

1 Nous sommes régis par des frontières. Les plus visibles sont terrestres et ont été mises en place pour délimiter les différentes souverainetés étatiques. Ces lignes créées par l'homme séparent deux ou plusieurs espaces différents mais elles sont plus que de simples limites : elles sont emplies d'histoires. Elles sont des réservoirs d'émotions, de souvenirs et de peurs. Certaines ont été éprouvées par les guerres, d'autres sont devenues des «no go zone" mystiques, des territoires en perdition ou des terres d'accueil. Nous en traversons fréquemment sans connaître leurs histoires. De cette frontière en émane une autre: la frontière émotionnelle. En effet, franchir une frontière terrestre est une épreuve physique mais au-delà, c'est une expérience émotionnelle: quitter son pays ou sa famille, devoir s'adapter, devenir une autre personne, se recréer. Dans cet article nous nous intéresserons à ces deux sortes de frontières à travers le personnage de Willy, membre d'une mara dans le film Sin Nombre de Cary Joji Fukunaga. Nous dresserons tout d'abord un bref contexte des maras, ces gangs qui sont le fruit de la guerre civile salvadorienne et des déportations américaines pour, par la suite, nous centrer sur l'itinéraire et la quête identitaire de Willy dans le 
film. Nous nous intéresserons à la dualité du personnage porteur d'un double prénom : Willy son identité civile et Casper son identité de membre de gang, ce que cette dualité amène dans cette quête identitaire et dans la traversée de ses frontières. Nous continuerons par l'analyse des relations que le personnage entretient avec le monde qui l'entoure et son incapacité à s'émanciper de son identité de marero.

\section{Le Salvador, bref contexte historique}

2 Dans son ouvrage Un Destin Ambigu: les illusions et les ravages de la politique étrangère américaine de Monroe à Reagan ${ }^{1}$, le journaliste et historien T. D. Allman analyse et critique la politique étrangère américaine, qui soutient des dictatures et entrave la liberté et les droits de l'Homme. Dans son passage sur le Salvador, il déclare :

On pouvait conduire pendant des heures sans jamais voir d'enfant porter des livres mais les hommes armés étaient partout, arrêtant les automobiles, surveillant les buvettes, observant ceux qui osaient aller à la messe, acheter le journal ou parler à un étranger. Dans ce pays tropical inondé de soleil l'ombre froide de la peur était partout ${ }^{2}$.

3 Entre 2 et 3 millions de Salvadoriens ont essayé de fuir cette « ombre froide » dans les années 70 en migrant vers d'autres pays dont les Etats-Unis et c'est de cette migration que les maras sont nées.

4 Le massacre des paysans salvadoriens en 1932 est l'un des premiers évènements déclencheurs des dynamiques de violence au sein de la population ${ }^{3}$. Maximiliano Hernandez Martinez, président du Salvador, est critiqué par sa population pour ses abus de pouvoir et les inégalités notables entre la classe paysanne, les gens du peuple, et l'oligarchie qui détient les terres ${ }^{4}$. Le gouvernement militarisé est allié avec los catorce, un groupe de 14 familles qui détient tous les droits sur les terres arables du pays et qui exploite ses employés, ce sont les principes du latifundisme ${ }^{5}$. Les paysans décident de se rebeller, et de cette rébellion naît un mouvement communiste, le PCS (Parti communiste du Salvador) dont Farabundo Marti ${ }^{6}$ est l'un des piliers. Ils présentent une liste de candidats pour les élections suivantes, qui sont truquées, ce qui les amène à mettre en route leur action armée : à la fin du mois de janvier 1932, ils s'unissent et attaquent les forces gouvernementales. En juillet, cette répression prendra fin avec un armistice de l'Assemblée législative du Salvador qui punit toute sorte de crimes contre la population. Ce massacre aura fait entre 10000 et 40000 victimes.

5 La révolte paysanne refera surface en 1979 et activera une guerre civile qui durera 13 années. Le Salvador est en difficulté : son gouvernement est toujours corrompu et le pays connaît un accroissement de sa population mais n'a pas assez de terres (los catorce détiennent la plupart des terres pour la culture du café et ne veulent pas les céder) ${ }^{7}$. Cette révolte se déclenche avec un putsch et le renversement du président, le général Romero, puis une junte se créée pour gouverner avec des représentants militaires mais aussi des civils. Ce premier putsch amène la création de groupes de protection et de revendication qui prennent en tant que modèle le PCS. Le FDR (le Front Démocratique Révolutionnaire) ou encore le Front Farabundo Martí de Libération (qui rend hommage à Farabundo) s'unissent avec les autres mouvements - il y en a 5 au total - et ces groupes révolutionnaires de guérillas marxistes ${ }^{8}$ sont rapidement rejoints par tout le clergé catholique. Face à cette guerre et devant l'impuissance d'un peuple, l'archevêque 
Romero, une figure religieuse du Salvador, appelle à l'aide le président Carter ${ }^{9}$ qui décide de suspendre l'aide que le gouvernement américain verse au Salvador; l'archevêque sera assassiné en pleine messe $^{10}$. Le Major Robert d'Aubuisson, commanditaire de cet assassinat, est un ancien agent des renseignements formé aux USA, il a aussi été leader des Escadrons de la mort ${ }^{11}$. Ces derniers sont responsables de la mort de l'archevêque Romero ${ }^{12}$ mais aussi les auteurs de plusieurs massacres dans des villages du Salvador comme celui d'El Mozote ${ }^{13}$. Le gouvernement salvadorien a pour but de faire taire sa population, de la purger des militants gauchistes, des jésuites et des communistes. C'est en 1992 après deux années de négociations ${ }^{14}$ que des accords de paix entre une guérilla d'obédience marxiste épaulée par d'autres pays d'Amérique Latine (Nicaragua et Cuba) et le gouvernement soutenu par les USA ${ }^{15}$ sont signés. Cette guerre aura fait 75000 morts $^{16}$.

\section{La création des maras}

6 Quand la communauté salvadorienne arrive aux Etats-Unis, à la fin des années $1970^{17}$ pour échapper à la guerre et aux conditions de vie difficile, elle vit un choc des cultures et l'adaptation est laborieuse. Les Salvadoriens décident de créer des groupes d'entraide pour défendre leurs droits - individuels et collectifs - en tant qu'immigrés et pour faire face aux violences au sein de leur quartier. La première mara, la Barrio 18, voit le jour dans les années 40 quand des jeunes de la bande mexicaine Clanton Street 14 - un des plus anciens gangs mexicains de Los Angeles - décide de quitter cette bande pour s'installer dans la rue 18 près de Rampart (à Los Angeles également) ${ }^{18}$. Dans les années 80, ce gang devient l'un des plus influents de Los Angeles et c'est à ce moment que la MS 13 naît, sous le nom Mara Salvatrucha Stoner. À l'origine, ce sont des fans de heavy métal qui se rencontrent pour partager leur passion mais aussi pour pouvoir échanger sur les problèmes qu'ils rencontrent. Ce groupe agit comme soutien moral et émotionnel face à la détresse de cette communauté et ce concept de bande se transforme progressivement : ils reproduisent et adaptent des comportements d'autres gangs en intégrant leur propre culture. Faire partie d'un gang c'est avoir des semblables, une famille qui est prête à protéger les siens. Graduellement, les deux gangs commencent à se faire une guerre de territoire puis se battent pour le monopole des trafics (extorsion, drogues, armes, humains) mais c'est aussi une guerre ethnique ${ }^{19}$, dans la 18 les membres sont issus de différents pays d'Amérique Centrale alors que la MS13 est constituée uniquement de Salvadoriens. Après la déportation de milliers de Salvadoriens vers leur pays d'origine suite à la mise en place d'une réforme des lois sur l'immigration ${ }^{20}$ - qui a pour but de déporter les personnes illégales qui ont été condamnées à un an de prison - ces groupes s'imposent dans la région mésoaméricaine.

Du côté du Salvador, on a peu de connaissances concernant ces groupes et le gouvernement n'est pas prêt à s'y intéresser, préoccupé par la guerre qui vient de se finir et par l'avenir du pays ${ }^{21}$. Certains membres de gang se retrouvent dans un pays qu'ils ne connaissent plus. L'ayant quitté très jeune, ils n'ont pas les repères qu'ils avaient aux États-Unis et il est difficile pour eux de s'adapter ${ }^{22}$. Ils préfèrent donc rester " entre eux », avec les membres de leur groupe. Par ailleurs, ils sont aussi vus d'une manière idéalisée par la population jeune: ils apportent une culture étatsunienne différente dans leur façon de s'habiller, de se comporter et dans la musique qu'ils écoutent. Ils présentent le gang comme un tremplin social, une sécurité financière et 
surtout une famille. Ce concept de «famille» leur permet d'endoctriner une jeunesse souvent sans repères ${ }^{23}$. Les violences familiales et sociétales restent difficiles à comprendre et à analyser pour un jeune, qui trouvera dans la mara une nouvelle famille et des réponses à ses questionnements. Ces bandes sont des microsystèmes, et comme l'explique Maria Santacruz ${ }^{24}$, il y a une hiérarchie dans chaque système. Le modèle de famille mis en avant n'empêche pas les maras d'être de véritables microsociétés qui arborent le même fonctionnement qu'une dictature ${ }^{25}$. Ces bandes, qui furent désorganisées à leurs débuts sont devenues, aujourd'hui des bandes criminelles qui se partagent le pouvoir, de la répartition des quartiers d'une ville aux relations des détenus de gangs dans les prisons. Cette hiérarchie intègre également une dimension genrée avec une reproduction de la division par sexe de la société, comme le montre le premier rituel d'entrée dans une mara appelé «brincada » ou «jumping in». Ce rituel a une dimension temporelle de résistance. Ainsi la recrue se fait rouer de coups pendant 13 secondes pour la MS13 ou 18 secondes pour la 18 th. ${ }^{26}$ Sur cet aspect, il faut souligner que les femmes ont le même choix que les hommes, cependant elles peuvent également "opter" pour l'option du viol par plusieurs membres du gang. Les femmes qui choisissent la deuxième option resteront les propriétés sexuelles des membres de gang alors que celles qui choisissent de se faire battre seront traitées avec « un peu plus de respect » et pourront essayer de gravir les échelons même si cela n'est pas fréquent. Une fois passés tous ces rituels, le marero ou la marera est présenté.e aux membres de son gang et on lui explique les règles à suivre : accepter cette identité collective et oublier son individualité. L'entrée en gang est violente, les rituels sont d'une brutalité inouïe. Une fois intégré, il est presque impossible d'en sortir. Cette impossibilité de sortie, ces frontières qui séparent la société et le cheminement des mareros sont au cœur du film Sin Nombre. Dans cette fiction, le protagoniste, Willy/Casper, franchit continuellement des frontières. Ces frontières qui sont d'abord physiques, se transforment rapidement en obstacles humains, sociétaux mais aussi identitaires :

D'un côté les êtres de la représentation sont des êtres fictifs, relevés de tout jugement d'existence, soustraits donc à la question platonicienne sur leur consistance ontologique et leur exemplarité éthique. Mais ces êtres fictifs n'en sont pas moins des êtres de ressemblance, des êtres dont les sentiments et les actions doivent être partagés et appréciés ${ }^{27}$.

\section{La frontière en tension : la dualité Casper/Willy}

Sin Nombre, de Cary Joji Fukunaga réalisé en 2009 est un film qui traite le sujet de la migration à travers le parcours d'un marero, Casper, mais aussi d'une jeune Hondurienne, Sayra. Casper, membre de la MS13, fait partie de la fraction de Tapachula au Mexique, alors que Sayra vit au Honduras. Elle est recherchée par son père - qui vient d'être déporté des Etats-Unis - qui souhaite la ramener avec lui, malgré elle. Casper est un personnage mystérieux, on ne connaît presque rien de lui, ni son âge, ni sa situation familiale, ni la façon dont il est entré dans cette mara. Durant la première partie du film, il est proche de deux personnes: Benito qu'il va prendre sous son aile, un enfant de son quartier qui idéalise les mareros et qui deviendra par la suite Smiley, et Martha Marlen, sa petite copine. Ses relations avec les membres du gang transposées à l'écran sont cordiales, il ne se mélange pas avec eux. Il disparaît souvent, d'où l'origine de son surnom Casper, du nom du fantôme du dessin-animé éponyme. ${ }^{28}$ 
9 La première frontière franchie dans le film est celle entre les genres cinématographiques. En effet, Sin Nombre est une fiction qui reprend des codes narratifs et esthétiques du documentaire. Dans son processus de création, le réalisateur a passé plusieurs mois à étudier les frontières et l'espace parcouru par des groupes de migrants, afin de pouvoir les transposer de manière réaliste dans son film. Pour cet ancrage dans la réalité, Fukunaga a choisi des acteurs professionnels mais a eu recours à des amateurs avec cette idée de brouiller encore une fois cette frontière entre la fiction et le documentaire. Les frontières nous sont montrées par le bais des images, c'est-à-dire, des paysages et des espaces dans le film mais aussi par des repères topographiques au bas de l'écran à chaque changement de frontières ${ }^{29}$. Avec ces repères, le réalisateur nous offre une vraie cartographie des frontières que les migrants doivent franchir pour se rendre aux Etats-Unis ${ }^{30}$.

Nous avons compté jusqu'à dix frontières terrestres tout au long du film,

1. son quartier la Bombilla, Mexique ${ }^{31}$

2. le quartier de Martha Marlen Market Hill

3. l'Immigrant rest stop (un endroit où les migrants se reposent, mangent et se douchent en attendant que le train redémarre)

4. Veracruz Highlands (les hauts plateaux de la ville de Veracruz)

5. Highlands above Mexico (les hauts plateaux en dessous de la ville de Mexico)

6. La Lecheira une station de train dans la municipalité de Tultitlán

7. la maison de Tia (une femme qui fait partie du même gang)

8. Mexican highway (l'autoroute mexicaine)

9. Reynosa une ville frontière des Etats-Unis dans l'Etat de Tamaulipas, Mexique

10. Rio Grande le fleuve qui sépare le Mexique des USA ${ }^{32}$.

11 Une des premières frontières filmiques qui nous est montrée se trouve dans l'ouverture du film. La séquence commence avec un plan large où l'on voit une forêt, la caméra est fixe et nous donne l'impression d'être au cœur de la nature. L'illusion est rapidement brisée par un plan américain sur un jeune homme torse nu qui fume. Le regard vide, il est assis sur une chaise. La caméra continue d'avancer vers lui. Nous nous rendons compte que nous ne sommes pas face à une forêt mais devant un poster dans une pièce d'appartement. La caméra dévoile un tatouage sur son dos : MS. Il marche vers cette affiche mais la contourne en baissant la tête. Le personnage se trouve enfermé dans une sorte de huis-clos, pris au piège dans cette pièce et dans son propre corps avec cette envie d'être ailleurs (la forêt). Cette scène introduit les questions qui se posent au personnage principal tout le long du film : l'envie de liberté, symbolisée par l'affiche de la forêt, et sa réalité entre quatre murs, son appartenance définitive au gang, signalée par le tatouage qu'il ne peut effacer.

En outre, le spectateur peut éprouver la réalité du personnage lors de sa traversée de diverses frontières physiques, qui le mettent face au danger et face aux autres. Pendant la première partie du film, à plusieurs reprises, Casper sort de son quartier La Bombilla pour aller à Market Hill, le quartier de son amie Martha Marlen, dans lequel il n'a pas le droit de se rendre, car c'est celui des rivaux de sa mara. Dans Market Hill, Casper n'est jamais «sur terre»: il doit à chaque fois gravir un mur pour arriver dans l'appartement de Martha Marlen, ou une tour pour pouvoir profiter d'un moment avec elle. La caméra suit le corps de Casper pour mettre en lumière celui de Willy, le citoyen $^{33}$. La prise de hauteur et les moments qu'il passe avec sa petite amie sont les 
seuls où il peut être sincère et se mettre à rêver de nouveau. Il redevient Willy, un jeune homme qui aimerait s'enfuir au Texas avec Martha. Cette hauteur lui permet aussi de se dérober des yeux de sa mara et donc de protéger sa petite amie de ceux-ci.

Aussi, Willy se heurte-t-il à deux obstacles : d'une part aux autres dans le but d'exister et d'autre part à sa propre identité. La frontière et la dualité des identités sont ancrées dans ce personnage. Casper le marero pour sa mara devient Willy pour Martha Marlen sa petite amie. On peut voir le protagoniste souffrir de cette dualité quand Lil Mago, son chef, lui demande des comptes et qu'il aimerait qu'il soit plus actif dans le gang. Sa double vie est éprouvante également avec Martha qui lui demande souvent ce qu'il fait avec ses amis, elle doute de sa sincérité dans la mesure où elle ignore qu'il appartient à un gang. Les deux personnages, Lil Mago et Martha, sont face à l'apathie de Willy. Cependant cette passivité va tomber en même temps que ce mur qu'il impose aux autres après la mort de sa petite amie Martha assassinée par Lil Mago qui essayait de la violer. Il sera animé par la vengeance, et par l'envie de reconquérir son identité d'origine. La scène du meurtre de Lil Mago son chef est emblématique de cette reprise d'identité. Cette séquence est déterminante. Elle se déroule sur le toit du train où Sayra se trouve avec son oncle, son père ainsi que d'autres migrants. Lil Mago, Smiley et Casper sont en mission d'extorsion, le train est à l'arrêt à cause du temps nuageux et pluvieux, les migrants sont cachés sous des bâches pour se protéger. Dans les premières séquences de ce film, le chef est dépeint comme étant le leader menaçant, il est souvent filmé en contre-plongée pour accentuer sa position de dominant, une technique bien connue dans la réalisation de films. Le montage alterné dans cette scène nous expose la façon dont les trois mareros occupent l'espace. Smiley suit les pas de son chef alors que Casper est en retrait. À travers les plans de cette scène on observe la domination sans partage du chef sur ses recrues : les plans larges dans lesquels il fait des signes de mains pour leur parler ou leur donner des ordres mais aussi avec des plans rapprochés. Le train est à l'arrêt, Casper participe passivement à cette mission jusqu'à ce que Lil Mago rencontre Sayra. Le montage alterné rythme la scène et accroît cette tension que l'on peut ressentir entre les personnages. Quand son chef lui demande de tenir la jeune fille pour qu'il puisse la violer, Casper a l'air impuissant face aux cris de celle-ci, au désespoir du père, à la violence qui l'entoure. Le train va redémarrer et c'est à ce moment que la caméra nous montre Casper dans un plan rapproché, il tranche la gorge de son chef et donc devient celui qui domine. Cette scène d'assassinat est précédée d'une série de contrechamps qui mettent en relief la confrontation entre les deux hommes. Casper reprend le pouvoir et peut redevenir Willy. La scène est rythmée par des sons diégétiques: les cris de tous les personnages mais aussi en son extradiégétique, une musique mouvementée - avec la présence d'un instrument à corde qui joue crescendo quand Casper est sur le point d'assassiner son chef - qui met en place une atmosphère vengeresse et libératrice pour le personnage principal. La scène du meurtre se termine par un échange de regards entre les deux hommes. Après l'acte commis, il se met à genoux, la pluie tombe. La violence de la pluie qui tombe se rapporte à la tempête intérieure de Willy, de ses différentes identités qui s'affrontent. Cette scène marque le début de la quête identitaire du protagoniste. Il ne se présentera plus en tant que Casper, il redevient Willy et Sayra sera la première à faire sa connaissance. Durant tout le film, Willy va essayer de se défaire de cette identité de marero. Cependant, conformément aux usages des gangs, on ne peut quitter ainsi la mara. Willy va tenter de franchir les frontières physiques et intérieures qui freinent son envie de s'échapper. Il retrouve son identité civile mais pour les gens qui l'entourent, il 
restera toujours un marero. Les personnes que Willy rencontre veulent sa mort. À plusieurs reprises, on essaie de le tuer: les membres de sa mara, les migrants sur le train qui attendent qu'il s'endorme ou encore des personnes qu'il rencontre brièvement, qu'il ne connaît pas mais qui reconnaissent ses tatouages et donc son appartenance à cette mara. Sayra est la seule personne qui s'intéresse à Willy malgré le désaccord de son père ${ }^{34}$. Pourtant elle ne suffira pas à le sauver.

\section{"Lil Mago, Willy s'en tirera pas » ${ }^{35}$. La frontière identitaire ou le point de non-retour} posture et la manière dont la caméra le filme, il occupe l'espace avec une distance, il est toujours en retrait ou essaie de fuir le rapprochement. Les deux personnages sont souvent filmés en plan large, ce qui instaure une frontière qui les sépare. La première scène dans laquelle les deux personnages ont une discussion est celle où ils sont en train d'attendre que le train redémarre. Willy s'éloigne dans un immeuble abandonné, Sayra décide d'aller lui parler. Ils échangent des banalités, quand Sayra s'approche de lui, il s'éloigne, la caméra les suit en travelling et toujours en plan large ou plan mimoyen. Willy ne fait pas partie du monde de Sayra, il ne fait partie du monde de personne, il est seul face aux autres.

Une scène qui est représentative de cette confrontation constante entre Willy et les autres, est celle où il se trouve dans un centre pour migrants. Willy est pris à parti par un homme qui n'accepte pas de le voir rester dans le centre. Les deux hommes, en plan taille, discutent, l'homme lui chuchote qu'il ne veut pas de racaille dans ce lieu, Willy rétorque en lui disant qu'il n'est plus un marero. Son identité de marero est celle qui le définit malgré son envie de s'en détacher, les autres qui l'entourent lui imposent une violence qu'il essaie de fuir, ils n'acceptent pas son choix de changer.

Une des scènes qui marque la fin de sa dualité identitaire Willy/Casper est celle dans laquelle il est face à son reflet dans un miroir. Il est avec Sayra dans une voiture pour franchir une des dernières frontières terrestres du film. Il regarde les vidéos qui lui restent de Martha Marlen sur sa caméra. Un montage alterné nous dévoile son visage et les images qu'il regarde. Il s'observe dans le rétroviseur intérieur, la caméra est figée sur son visage et sa main qui essaie, d'effacer - en grattant - le tatouage en forme de larme qu'il a en dessous de l'œil. La culture de gang peut être décrite comme « un univers de significations partagées, transmis de génération en génération, qui inclut des symboles et des signes de reconnaissance, des règles et des rituels, de même que des normes et des valeurs $\aleph^{36}$. Les tatouages comme marqueurs d'appartenance sont très importants dans les gangs au Salvador ainsi qu'aux États-Unis ${ }^{37}$. Ils font partie de l'apparence des mareros et sont la trace de leur engagement. Un marero est obligé de se tatouer en tout premier lieu le nom de son gang. Certains tatouages font référence aux rangs franchis, aux meurtres commis, ou à la vie qu'ils mènent, d'autres auront des significations spécifiques : la toile d'araignée est liée au pouvoir et à l'expansion, les trois points pour décrire la «vida loca » ou encore des tatouages religieux pour demander pardon ${ }^{38}$. Les tatouages sont aussi un reflet de l'expérience du marero. Plus un marero a de tatouages, plus il impose le respect, car ce sont des preuves de son évolution dans le gang ${ }^{39}$. Même si les tatouages peuvent être effacés, leurs traces persistent. Ces marqueurs d'appartenance deviennent une sorte d'enfermement pour 
les mareros qui ne peuvent pas s'en défaire comme nous pouvons le voir avec Willy. Cette larme qu'il essaie d'effacer est la métaphore de son passé et est une frontière qu'il n'arrive plus à dépasser. Malgré ses essais, sa persévérance dans le franchissement des frontières physiques et identitaires, Willy a atteint un point de non-retour.

Le voyage identitaire de Willy prendra fin quand il franchira la frontière ultime, celle de la mort. Cette séquence se déroule en plusieurs scènes. La première est ouverte par un plan large de Sayra et Willy qui se dirigent vers la rivière pour pouvoir passer du côté de la frontière étatsunienne. Willy, Sayra et le passeur marchent dans de hautes herbes. Willy se sent observé, une série d'images alternées nous révèle des hommes dans les hauteurs, dos au soleil, leurs visages ne sont pas visibles. Une série de plans saccadés entre Willy et les hauteurs traduisent que la mara est à ses trousses. Willy demande au passeur et à Sayra d'aller plus vite. Dans un autre plan large, avec en toile de fond le Rio Grande - le fleuve qui sépare les Etats-Unis du Mexique - Willy doit payer le passeur et décide de lui donner la caméra. Le seul objet qui lui reste avec les derniers souvenirs qu'il a partagés aux côtés de Martha Marlen comme signe d'un détachement de son passé. Willy sera assassiné par Smiley, la jeune recrue dont il était le mentor. Ce moment fait écho à la scène du meurtre du chef par Willy, et referme la boucle montre dans laquelle les membres de gangs sont emprisonnés. Il a tué donc il sera tué un jour ou l'autre, il a commis l'irréparable : ôter la vie de son chef. On revoit le corps de Willy gisant dans un ruisseau qui est devenu rouge, Sayra est dans l'eau et regarde ce corps. Elle observe également son propre passé et le chemin qu'elle a accompli avant de franchir sa dernière frontière, celle qui la mène aux États-Unis. La musique qui l'accompagne "La Última Palabra", le dernier mot en français, une musique traditionnelle zapotèque raconte une histoire d'amour et les derniers mots d'un homme pour une femme ${ }^{40}$. Sayra va vers un avenir incertain mais elle est en vie et a réussi grâce à Willy, elle y est arrivée "par les griffes du diable et non les mains de Dieu $»^{41}$ tandis que Willy est mort des mains de sa familia.

\section{Conclusion}

Willy n'a pas réussi à franchir la frontière qui le séparait de sa rédemption. Il a dû faire face à des frontières physiques et identitaires. À travers la caméra, nous sommes exposés aux souffrances de Casper et à son incapacité à redevenir Willy ainsi qu'à la dureté du monde qui l'entoure. Les choix du réalisateur avec cette représentation de la mara et cette impossibilité de sortie ${ }^{42}$ sont empruntés au genre du réalisme et contribuent à ce que le spectateur ait de l'empathie pour Willy qui devient aussi une victime. Le film a permis au spectateur de franchir une frontière, celle dressée par la presse qui fait le portrait de délinquants, mais que le cinéma rend poreuse en individualisant les destins et déterminismes sociologiques ${ }^{43}$. Cette réalité et la limite entre la fiction et le documentaire que le réalisateur franchit à plusieurs reprises, dans ses choix esthétiques et narratifs, est choisie: le réalisateur nous aide à mieux comprendre les maras même si nous ne pouvons saisir ce phénomène complexe que superficiellement. Les représentations visuelles des gangs et des bandes criminelles sont souvent idéalisées et peuvent faire rêver les jeunes spectateurs d'une vie de hors la loi, une vie dans laquelle l'argent et le pouvoir vont de pair avec le danger ${ }^{44}$. Sin Nombre déconstruit la "glamourisation » de la vie en gang telle qu'elle est véhiculée par la culture populaire ${ }^{45}$. Le réalisateur parvient à nous exposer un sujet sensible sans 
artifices et avec clarté : la vie puis la mort d'un jeune marero qui se bat contre la dualité de son identité, les autres en tant qu'obstacles et les frontières qu'il doit dépasser pour exister. La fin durant laquelle le réalisateur a choisi un plan où l'on voit flotter le corps de Willy dans la rivière dessine en creux le sort de nombreux latino-américains morts dans le Rio Grande. La rivière est une frontière-cimetière, comme la Méditerranée l'est pour les migrants en provenance de régions sub-sahariennes. Les continents changent, mais les frontières meurtrières se répètent.

\section{BIBLIOGRAPHIE}

Kacou Elom Jean-Michel АDОВОЕ, Le front farabundo martí de libération nationale au Salvador : 1980-2009, Togo, Université de Lomé, 2010.

James BARGENT, « Hidden meaning of Honduras Mara Gang Tattoos Explained », InSight Crime, Septembre 2014 [consulté le 27 janvier 2020] <URL : https://insightcrime.org/news/brief/ hidden-meanings-honduras-mara-gang-tattoos/>.

Manuelle BÉRIAULT, Catherine LAURIER, Jean-Pierre GUAY, «L'adhésion à la culture de gang : le rôle de l'identité ethnique chez les jeunes contrevenants de Montréal et ses environs ", Criminologie $\mathrm{n}^{\circ} 50$ (2017), p. 287-310.

Robert CLARK et William MITCHELL, « Target-Centric Network Modeling: Case Studies », Analyzing Complex, Los Angeles, Editions Sage, 2015, p. 169-183.

Maya collomBon, « Maras, pandillas et autres outsiders. Pour une ethnographie des gangs latinoaméricains : Introduction ", Ethnographier les gangs : maras, pandillas et autres outsiders en Amérique Latine, Paris, Cultures \& Conflits Sociologie Politique de l'International $n^{\circ} 110 / 111$, L'Harmattan, 2018, p. 7-37.

Juan José MARTINEZ D’AUBUISSON, « La transformation des maras salvadorienne. Construire le pouvoir depuis ses marges ", Ethnographier les gangs : maras, pandillas et autres outsiders en Amérique Latine, Paris, Cultures \& Conflits Sociologie Politique de l'International, L'Harmattan , $\mathrm{n}^{\circ}$ 110/111 (2018), p. 141-156.

Timothy D. ALLMAN, Un Destin Ambigu : les illusions et les ravages de la politique étrangère, Paris, Éditions Flammarion, 1986.

Clément DETRY, «Soutien militaire étasunien au Salvador : George Bush père a eu des scrupules », Mediapart, Décembre 2016 [consulté le 25 janvier 2020] <URL : https://blogs.mediapart.fr/ clement-detry/blog/171216/soutien-militaire-etasunien-au-salvador-george-bush-pere-eu-desscrupules $>$.

Sophie DJIGO, L'éthique du gangster au cinéma, une enquête philosophique, Rennes, Presses Universitaires de Rennes, Collection Aesthetica, 2016.

Frédéric FAUX, Les maras, gangs d'enfants. Violences urbaines en Amérique centrale, Paris, Éditions Autrement Frontières, 2006.

Alain GANDOLFI, Les luttes armées en Amérique latine, Paris, Presses Universitaires de France, 1991. 
Quentin LIENARD, « La responsabilité de l'État pour les violations des droits de l'homme commises par des groupes paramilitaires devant les instances Inter-américaines » in Amérique latine de la violence politique à la défense des droits de l'Homme, Paris, L'Harmattan, 2012, p. 205-214

Oscar et Juan José MARTíNEZ, A History Of Violence: Living and Dying in Central America, Londres, Editions Verso, 2017.

Oscar et Juan José MARTíNEZ, El Niño de Hollywood : comment les USA et le Salvador ont créé le gang le plus dangereux du monde, Paris, Éditions Métailié, 2020.

Sarah MEKDJIAN, « Les apports du cinéma à une (géo-)graphie des frontières mobiles et des migrations frontalières ", Annales de géographie, $n^{\circ}$ 695-696 (2014), p. 784-804.

Dario MORENo, U.S Policy in Central America, The Endless Debate, Floride, Florida International University Press Miami, 1990.

Gilberto OROzco, Daniel C. Pineda y «la última palabra», Corta Mortaja, 2016 [consulté le 3 février 2020] <URL : http://cortamortaja.com.mx/arte-cultura-istmo/articulos/2559-daniel-c-pineda-yla-ultima-palabra>.

Jean PIEL, «L'expropriation des terres et la formation du latifundium en Amérique espagnole et latine », HISTOIRE(S) de l'Amérique latine, vol. 8 (2013), p. 1-14.

Jacques RANCIÈRE, Le Destin des images, Paris, La Fabrique éditions, 2003.

RACHID, « Génération Scarface, la place du trafic dans une cité de banlieue parisienne » in Déviance et Société, vol. 28 (2004), p. 115-132.

MONSIEUR X, La guerre civile au Salvador, Rendez-vous avec Monsieur X, France Inter, Janvier 2017 [consulté le 01 janvier 2020] < URL : https://www.franceinter.fr/emissions/rendez-vous-avec-x/ rendez-vous-avec-x-20-janvier-2007 >.

\section{NOTES}

1. Timothy D. ALlman, Un Destin Ambigu : les illusions et les ravages de la politique étrangère, Paris, Éditions Flammarion, 1986.

2. Ibid.

3. «Au Salvador, le point de départ officiel de la violence actuelle remonte à ces journées de janvier 1932 où l'armée massacra sans pitié 30000 paysans qui réclamaient une réforme agraire. » Alain GANDOLFI, "Les déchirements sanglants du Salvador» in Les luttes armées en Amérique latine, Paris, Presses Universitaires de France, 1991, p. 103.

4. « Since the early 1930's, the Salvadoran army, supported by a handful of powerful families, had ruled the country with little concern for basic human rights. » Dario MORENO, U.S Policy in Central America The Endless Debate, Floride, Florida International University Press Miami, 1990, p. 71.

5. «Ceci est le résultat d'une longue histoire agraire initiée par la Conquista et aggravée durant les 500 ans qui suivirent. Très tôt en effet, au lendemain de la conquête de l'Amérique, le pouvoir colonial a distribué des portions énormes de terres aux conquistadores ou à leurs héritiers, ne laissant au reste de la population... que des restes, justement. » Jean PIEL, "L'expropriation des terres et la formation du latifundium en Amérique espagnole et latine ", in $\operatorname{HISTOIRE(S)~de~}$ l'Amérique latine, vol. 8 (2013), p. 1.

6. Farabundo Martí est une figure importante dans l'histoire du Salvador. C'est après un exil au Nicaragua (dû à ses positions anti impérialiste), qu'il revient clandestinement au Salvador et met 
en place ce groupe. Mémoire universitaire de Kacou Elom Jean-Michel ADOBOE, Le front farabundo marti de libération nationale au Salvador : 1980-2009, Togo, Université de Lomé, 2010.

7. «Entre 1900 et 1985 la population a presque sextuplé. Contrairement à celle d'autres pays latino-américains, elle est essentiellement rurale ( $86 \%$ ), la plus massivement rurale avec celle du Honduras (90\%). C'est dire l'importance du problème de la terre.» Alain GANDOLFI, «Les déchirements sanglants du Salvador ", in Les luttes armées en Amérique latine, op. cit., p. 104.

8. Ibid p. 104-106.

9. Depuis le début de son mandat, Carter voulait aider les Salvadoriens contre leur gouvernement militaire oppressant. "Carter sought to devise a new regional policy that would stimulate reforms and reduce human rights abuses, while simultaneoulsy undermining the politcal support of the armed insurgent. » Dario MORENo, U.S Policy in Central America The Endless Debate, op. cit., p. 71.

10. «La preuve de l'existence d'instructions précises émanant d'organes de l'État entraîne fort logiquement l'attribution à l'autorité publique de l'acte ordonné. Une telle situation s'est notamment présentée dans l'affaire Monsignor Oscar Arnuflo Romero y Galdamez c.El Salvador.» Quentin LIENARD, «La responsabilité de l'État pour les violations des droits de l'homme commises par des groupes paramilitaires devant les instance Interaméricaines » in Amérique latine de la violence politique à la défense des droits de l'Homme, Paris, L'Harmattan, 2012, p. 207.

11. Les escadrons de la mort sont un groupe de paramilitaires faisant partie de l'élite qui organise en secret des meurtres, des enlèvements de toute personne jugée nocive au bon fonctionnement du pays.

12. Carter après l'appel de l'archevêque a suspendu l'aide financière militaire que les USA versaient au Salvador ce qui a déplu aux escadrons et au gouvernement militaire salvadorien.

13. Clément DETRY, Soutien militaire étasunien au Salvador: George Bush père a eu des scrupules, in Mediapart (Décembre 2016) [consulté le 25 janvier 2020] <URL: https://blogs.mediapart.fr/ clement-detry/blog/171216/soutien-militaire-etasunien-au-salvador-george-bush-pere-eu-desscrupules >.

14. «Sous l'égide de l'ONU à Genève, de nouvelles négociations s'amorcent au début avril 1990 entre le gouvernement et la guérilla "en vue de mettre un terme au conflit armé, par la voie politique, dans les délais les plus brefs afin de promouvoir la démocratisation du pays, de garantir le respect intégral des droits de l'Homme et de réunifier la société salvadorienne". » Alain GANDOLFI, «Salvador: l'alternance des combats et des trêves", in Les luttes armées en Amérique latine, op. cit., p. 207-208.

15. La guerre civile au Salvador, Rendez-vous avec Monsieur X, France Inter, Janvier 2017 [consulté le 01 janvier 2020] <URL: https://www.franceinter.fr/emissions/rendez-vous-avec-x/rendezvous-avec-X-20-janvier-2007 >.

16. Ibid.

17. «La Mara Salvatrucha ou MS13 est fondée à Los Angeles (quartier Pico) par une bande d'adolescents salvadoriens, réfugiés arrivés dans les années 1970. " Maya CollomBon, "Maras, pandillas et autres outsiders. Pour une ethnographie des gangs latino-américains Introduction ", Ethnographier les gangs: maras, pandillas et autres outsiders en Amérique Latine, Paris, Cultures \& Conflits Sociologie Politique de l'International no 110-111 Ed. L'Harmattan, 2018, p.26.

18. «L'histoire de Barrio 18 commence dans les années 1940, lorsqu'un groupe de jeunes quitte une bande d'origine mexicaine Clanton Street 14. Ce groupe décide alors de s'installer rue 18, près de Rampart. Progressivement d'autres jeunes les rejoignent, pour la plupart originaires d'Amérique Centrale.» Juan José MARTINEZ D'AUBUISSON, «La transformation des maras salvadoriennes. Construire le pouvoir depuis ses marges ", Ethnographier les gangs : maras, pandillas et autres outsiders en Amérique Latine, Paris, Cultures \& Conflits Sociologie Politique de l'International n 110/111 Ed. L'Harmattan, 2018, p. 142-143. 
19. «Les deux gangs deviennent bandes ennemies dans les rues de Los Angeles à la fin des années 1980. Leur conflit est connu pour être une "lutte à mort" ou l'anéantissement de l'autre est un axe directeur de l'union et produit l'intensité du sentiment d'appartenance. » Maya CoLlombon, «Maras, pandillas et autres outsiders. Pour une ethnographie des gangs latino-américains Introduction ", Ethnographier les gangs, op. cit., p.26.

20. «En 1996, à la suite de la réforme des lois sur l'immigration illégale et la responsabilité migratoire, le congrès américain approuve la déportation de tout citoyen non étasunien condamné à plus d'un an de prison. C'est ainsi que près de 50000 prisonniers - dont de nombreux membres des maras - et 160000 migrants sans papiers sont déportés dans la décennie suivante, en majorité au Salvador, au Guatemala et au Honduras. ", Maya CoLLOMBon, « Maras, pandillas et autres outsiders. Pour une ethnographie des gangs latino-américains Introduction", Ethnographier les gangs, op. cit., p. 26.

21. "Ceux qui échouent au Salvador, au Honduras ou au Guatemala découvrent alors un paradis pour délinquants aguerris où la police est impuissante, où les prisons débordent, où les armes circulent, et dont ils sont les nouveaux maîtres. Le contexte de violence militaire, économique et sociale dans lequel baigne l'Amérique centrale sera le terreau sur lequel la culture de gang va s'épanouir. ", Frédéric FAUX, Les maras, gangs d'enfants. Violences urbaines en Amérique centrale, Paris, Éditions Autrement Frontières, 2006, p. 51.

22. «Et ils sont beaucoup à avoir débarqué comme ça, sans très bien savoir si leurs haines les plus ancrées seraient encore pertinentes dans ce pays où ils étaient nés mais qu'ils connaissent à peine. » Oscar et Juan José MARTÍNEZ, El Niño de Hollywood: comment les USA et le Salvador ont créé le gang le plus dangereux du monde, Paris, Éditions Métailié, 2020, p. 96.

23. «Pour les jeunes Salvadoriens, victimes de la pauvreté, sans éducation, sans perspectives d'avenir ni travail, ils sont apparus comme des dieux. Une fascination que les deportados se sont appropriée pour assurer leur emprise sur les esprits et les comportements, imposer "un sentiment d'appartenance, une culture de la violence systématique qui conditionnent la vie pour toujours". ", Frédéric FAUX, Les maras, gangs d'enfants, op. cit., p. 31.

24. María Santacruz Giralt est une psychologue salvadorienne qui en 2001 publie ses travaux sur les membres de maras, elle apparaît dans l'ouvrage de Frédéric FAux dans le chapitre "Qui sontils ? Les réponses de María Santacruz Giralt », Les maras, gangs d'enfants, op. cit., p. 26-33.

25. « Tous les pandilleros peuvent parler, mais il n'y en a qu'un qui décide. La mara est un groupe ultra-hiérarchisé. La décision du chef est un fait qu'on ne discute pas. » Frédéric FAux, Les maras, gangs d'enfants, op. cit., p. 31.

26. Nous pouvons voir ce rituel dans le film quand la recrue Smiley entre dans le gang à partir de la $3^{\mathrm{e}}$ minute.

27. Jacques RANCIÈRE, Le Destin des images, Paris, La Fabrique éditions, 2003 p. 132.

28. Les membres de gang ont souvent des surnoms qui les caractérisent dans ce film, cela est représenté par Casper mais aussi Benito qui sourit après avoir réussi le rituel d'entrée dans la mara ce qui lui vaut son surnom Smiley.

${ }^{28}$ Interview du réalisateur dans le DVD Sin Nombre.

29. Sarah MEKDJIAN, «Les apports du cinéma à une (géo-)graphie des frontières mobiles et des migrations frontalières ", in Annales de géographie, $\mathrm{n}^{\circ}$ 695-696 (2014/1-2), p. 84-804.

30. Ibid.

31. Tous les quartiers cités se trouvent au Mexique.

32. C'est en lisant le scénario et en regardant le film que ce chemin a été tracé.

33. Nous utilisons ici le terme citoyen car dans les maras, le prénom de naissance n'est qu'un prénom civil et n'a plus de sens, il doit être détaché du membre de la mara.

34. Horacio, le père de Sayra, à plusieurs reprises lui interdit de lui parler car il est dangereux et est le présage de mauvaises choses. 
35. Le message sur le mur que Willy aperçoit en sortant de chez Tia à 1 heure et 8 minutes dans le film.

36. M. BERIAULT, C. LAURIER et J.-P. GUAY, «L'adhésion à la culture de gang: le rôle de l'identité ethnique chez les jeunes contrevenants de Montréal et ses environs ", Criminologie ${ }^{\circ} 50$ (2017), p. 287-310.

37. «Gangs members directly from El Salvador generally have more prominent tattoos than those who become members in the United States. » Robert Clark et William Mitchell, "TargetCentric Network Modeling: Case Studies ", Robert CLARK et William MITCHELL, Analyzing Complex, Los Angeles, Editions Sage, 2015, p. 172.

38. James BARGENT, « Hidden meaning of Honduras Mara Gang Tattoos Explained », InSight Crime, Septembre 2014 [consulté le 27 janvier 2020] <URL : Hidden Meanings of Honduras Mara Gang Tattoos Explained - InSight Crime>.

39. «[...] an indication of the degree of gang involvement with older or more active members wearing the larger, more prominent tattoos. ", Robert Clark et William Mitchell, « Target-Centric Network Modeling: Case

Studies ", Robert Clark et William Mitchell, Analyzing Complex, Los Angeles, Editions Sage, 2015, p. 177.

40. Gilberto orozco, Daniel C. Pineda y «la última palabra», Corta Mortaja, 2016 [consulté le 3 février 2020] <URL : http://cortamortaja.com.mx/arte-cultura-istmo/articulos/2559-daniel-c-pineda-yla-ultima-palabra>.

41. Cette citation est tirée d'une des scènes du film quand les deux personnages attendent le train et discutent. Sayra explique à Willy qu'une de ses voisines est voyante et lui aurait dit qu'elle allait réussir son voyage jusqu'aux Etats-Unis non pas par la grâce de Dieu mais par les griffes du Diable, à partir de la $54^{\mathrm{e}}$ minutes dans le film.

42. Dans son ouvrage, Les Maras gang d'enfants. Violence urbaine en Amérique Centrale, Frédéric Faux a interviewé un ancien marero qui pour s'en sortir explique qu'il faut soit se marier soit devenir chrétien. Et que même si on choisit de sortir de ce système, cela prendra des années pour prouver qu'on a vraiment changé de vie et que le choix pris est le bon et que ce n'est pas une trahison, p. 114-115.

43. "D’un côté les êtres de la représentation sont des êtres fictifs, relevés de tout jugement d'existence, soustraits donc à la question platonicienne sur leur consistance ontologique et leur exemplarité éthique. Mais ces êtres fictifs n'en sont pas moins des êtres de ressemblance, des êtres dont les sentiments et les actions doivent être partagés et appréciés. » Jacques RANCIÈRE, Le Destin des images, op. cit., p. 132.

44. Nous pouvons voir cette idéalisation dans l'article "Génération Scarface ", où l'auteur interviewe des jeunes de banlieues qui se sont inspirés de ce film pour créer leurs propres empires : RACHID, «Génération Scarface, la place du trafic dans une cité de banlieue parisienne » in Déviance et Société, vol. 28 (2004), p. 115-132.

45. "Les gangsters sont des figures séduisantes qui se sont affranchies du système, c'est-à-dire, de la moyenne ",

Sophie DJIGo, L'éthique du gangster au cinéma, une enquête philosophique, Rennes, Presses Universitaire de Rennes, Collection Aesthetica, 2016, p. 17. 


\section{RÉSUMÉS}

Les maras d'Amérique centrale ont toujours été un objet de fascination. Ces groupes dits ultraviolents et dangereux constituent des microsystèmes ayant leur propre fonctionnement à l'origine de leur construction identitaire. Les images et les imaginaires qui les entourent ont été le sujet de plusieurs études universitaires mais aussi de multiples créations artistiques, notamment audiovisuelles. L'image visuelle est un medium à la portée de tous, grâce auquel nous pouvons imaginer, créer et représenter le monde qui nous entoure. Il va de soi que, comme toute création, ces représentations dépendent d'un point de vue qui oriente la lecture du spectateur. Dans Sin Nombre du réalisateur américain Cary Joji Fukunaga (2009), nous sommes amenés à ressentir de l'empathie pour Willy, un ancien marero (membre de gang) qui veut fuir sa mara mais s'en trouve empêché. Cet article aura pour but d'étudier les frontières géographiques et émotionnelles que doit franchir le protagoniste pour se libérer de son identité de marero.

The Central America's maras have always been an object of fascination. These so-called ultraviolent and dangerous groups constitute microsystems with their own functioning at the origin of their identities's construction. The images and imaginaries that surround them have been the subject of several academic studies but also of multiple artistic creations, notably audiovisual. Visual art is a medium within everyone's reach, a medium in which we can imagine, create and represent the world surrounding us. It goes without saying that, like any creation, these representations depend on a point of view that guides the spectator's reading but also feelings. In American director Cary Joji Fukunaga's Sin Nombre (2009), we are confronted with this idea from a point of view that declares its empathy with Willy, a former marero (gang member) who wants to flee his mara but is prevented from doing so. This article will aim to study the geographical borders but also the emotional boundaries that the protagonist must cross to free himself from his identity as a marero.

\section{INDEX}

Mots-clés : cinéma, maras, identité, frontières

Keywords : cinema, maras, identity, borders/boundaries

\section{AUTEUR}

\section{SAFIA BOUFALAAS}

Université Grenoble-Alpes

The City University of New-York 